\title{
ChatApp with Encryption using Firebase
}

\author{
Pavankumar Patel \\ Department of Computer Science \\ Lakehead University \\ ThunderBay, Canada \\ ppate159@lakeheadu.ca
}

\author{
Ishani Bhadoria \\ Department of Computer Science \\ Lakehead University \\ ThunderBay, Canada \\ ibhadori@lakeheadu.ca
}

\author{
Jinan Fiaidhi \\ Department of Computer Science \\ Lakehead University \\ ThunderBay, Canada \\ jfiaidhi@lakeheadu.ca
}

\begin{abstract}
Communication and interaction between one another is becoming integral part of everyone's life. From small conversation to meetings in an multinational companies it is every difficult to live without communication.Initially, in former days when their where no mediums for communicating from distant places the only way of conversation was face to face meet-ups. As the generation changed and year passed the technologies gave human race the power to communicate overseas. Today, Those technologies are under high risk of getting hacked. To make sure the communication remains confidential we need to take serious steps. This research paper includes the details about an chat application application to send private and confidential instant messages without the fear of interference. A secure communication path is created with encryption protocol.

Index Terms-Android Studio, Encryption, secure communication, Authentication
\end{abstract}

\section{INTRODUCTION}

The use of social media is increased with the increase in population. In recent years, Chat applications have improved and made substantial improvements to the social media due to its distinctive characteristics, which attract audiences. It offers real-time messages and provides various services like text, images, data, etc. In addition, cross platforms including Android and iOS are supported. There are now 100 million mobile users who use monthly chat applications. Security is of utmost importance in chat applications but few take this seriously. It is very important to make more secure application for better communication. In, today's time where most of the chat application use Transport layer protocol for security. It is difficult to ensure that the data is secured. As, the service provider has the full access to all the message sent and received by their user.

In this paper, we focus mainly on security and confidentiality of user messages by proposing the end to end encryption. Our application make sure that the user message sent by sender is original and is not manipulated by third party.

\section{BACKGROUND}

\section{A. Instant Chat Applications}

1) Whatsapp: Whatsapp is one of the most used application for communicating. It provides the text message, image, video, audio sending features to the user. Whatsapp has recently introduced end-to-end Encryption. However, as whatsapp is private and not the opensource it is had to verify if it is really so as announced. Whatsapp claims that the message stored on their storage are in encrypted format. Neither, any one of the whatsapp employee can read the original data nor change manipulate it. They also claim that the images and videos on their storage are in encrypted form.

2) WeChat: This Chat application is leading China market. WeChat is the third popular messaging application in the market available in different platforms including iOS and Android. It supports sending voice, video, pictures and text messages. WeChat does not provide end-to-end encryption meaning that encryption methods that is used is based on public key encryption, but the user needs to trust the WeChat servers.

3) Viber: Viber provides intant text message and VoIP calls to the user. Using viber a person can call the other user who is not on viber, it cost few cents per minute. Viber provides End-to-End Encryption for text message in private and group but images and videos are stored in original format on their storage. Strangers add you to friends list without permission in Viber. Their local storage is insecure.

4) Facebook Messenger: Facebook messenger is most popular chat application. They give user the choice to use end to end encryption or not. General messages provide only TLS encryption. Facebook message's secret chat provide end to end encryption for text messages but they scan the images to check any cases of child abuse. If any such images are detected those are deleted immediately and reported.

\section{B. Security Service}

1) Confidentiality: When the messages are exchanged between two parties through a communication channel that should be readable only to the intended partied which is called confidentiality. This is achieved with encryption mechanism. A message is encrypted using cryptography techniques.This technique changes the look of the message to distract the attacker from getting the correct message.

2) Authentication: The most important part of the security aspect is authentication. Authetication is the process where system checks the identity of the user if he/she is a valid member or not. strangers or unauthorized persons are restricted through such systems from breaking in. For this purpose today various type of techniques are used. For checking if it is a user or a bot "captcha's " are used. For login purpose one-time- 
password(OTPs) are used. Even email verification are used at many place.

3) Integrity: Integrity deal with the originality of data. Hackers try to change the data and keep the original data with them. It is very important to know if the data sent by sender is the only message recieved by the reciever. For this purpose Hash map are used. Even if the hacker changes the message he/she won't be able to change the hash map. Hashmap value verifies and esure the reciever that the data is original.

\section{End-To-End Encryption}

The struggle of data security and privacy is a battle that is fought on many fronts, but at the end, it boils down to one thing that is: whenever a private data is send to another computer or server on internet, once the data packet is send in form of voice call, chat, email or credit card number over the jungle of internet, no control remains over who lays their hand on it. This is the nature of internet.

Data and voice packets pass through many unknown servers, routers, and devices where any hacker or rouge state agent can intercept them. Thus, encryption comes to picture. $\mathrm{E} 2 \mathrm{EE}$ is implementation of asymmetric encryption.

E2EE stands for end-to-end encryption. Its central idea is to prevent anyone in the middle from accessing private communications. Until recent times end-to-end encryption was sole domain of tech savvy because of complicated operations required to use it. However recent technologies have made it more accessible and easier. [3]

Use of E2EE ensures that when an email is sent or when we message to someone no one monitoring the network can see the content which is send. No hackers, no government and not even the company has that facilitates the communication can read the message. This is very different approach from the encryption that most companies where using already.

The approaches and techniques already in use protects the data in transit between particular device and the company's sever. Gmail and Hotmail is the example of such companies which can get content of your message because of lack of usage of E2EE encryption and having a hold on encryption keys. E2EE eliminates such cases because there is no decryption key with service provider.

Every user of E2EE has two keys. One is known as public key while another is known as private key. Both the keys are mathematically related encryption keys. Public key can be shared with anyone while private key as the name suggest is only known to user. While sending message sender uses receiver's public key to encrypt message and create a cipher text.

Further this message is sent on public network though it may pass through multiple servers it is impossible to convert cipher text to plaintext. Thus, after message reaches to the inbox of receiver it is converted to readable form. While replying same process is repeated by receiver by using sender's public key.

\section{RELATED WORK}

In 2013 Dec, Ali Makki Sagheer et al, proposed a solution that gives secrecy and uprightness to SMS data by applying a crossbred cryptographic plan which join the AES for encryption/unscrambling plan and RC4 for key extension and generation algorithms to satisfy all the more intense security issues. The proposed model is actualized by Java programming dialect in view of NetBeans platform. The proposed framework was tried on different cell phones, for example, the Nokia 5233. [1]

In 2014 May, H.C. Chen et al. exhibited another idea about Mobile Text Chat utilizing a revolution session key based transposition cryptosystem plan. Their proposed conspire just manages the safe content transposition for mobile chat framework. It acclimatized the technologies of classical block cipher, substitution and transposition. Also, the new session key can be created by the network pivot innovation. Itcould be easily applied to transmit via mobile devices using the quick encryption algorithm. [4]

In 2014 July, R.N. Akram et al, evaluated the security and privacy preserving features introduced the current mobile chat services. They additionally put advances a fundamental system for an end to end security and protection mobile chat service and related necessities. [3]

\section{Proposed Architecture}

The proposed model is the client server based android application with firebase as the backend database. User needs to be registered with the application to use it. Only User's name and email id is stored in the database. The messages are stored in the encrypted format.

\section{A. Security model}

1) The sender type Text Message (TM)

2) $\mathrm{TM}$ converted to Bytes Array (BA)

3) Encrypt the BA (EBA): performed by AES with the Generated secure key

4) Convert the EBA to String (ES)

5) Send the ES to the server

6) The recipient receives the ES

7) Convert the received ES to Bytes Array (EBA)

8) Decrypt the EBA (BA)

9) Convert the BA to string which is same the sender message (TM)

\section{B. Process Flow of Application}

Let have a glance over how the flow of the application. Their are many activity pages in this android application. Starting from the beginning once the user have install the application he/she will see the page with option to login or register. 
Following is the Flow diagram of Application GUI. It doesn't included the Encryption Part as it is part of background where all the magic happens.

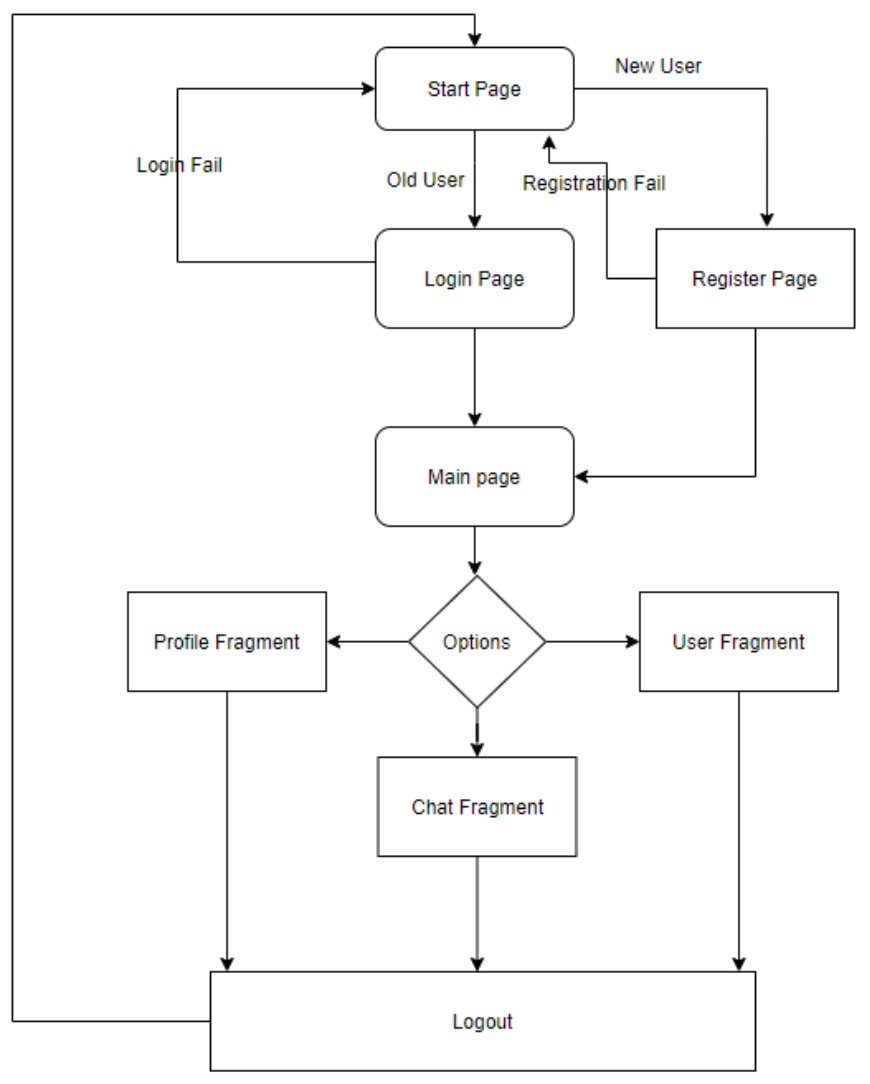

Fig. 1. Flow Diagram

\section{Backend Connection}

As we know every user interactive application needs a back-end cloud storage or database.For Our Chat Application we have used Google's firebase for building the database. Their are various methods available but the firebase is the easiest and developer friendly enviroment to create database.

To create firebase database and link it with the application we need to create the project on the firebase. Following are the steps to create and link the dataset to the android studio ide.

1) Create Firebase project ${ }^{[2]}$

2) Download the .JSON file created

3) Store that JSON file in the App directory of the project.

4) Now, login with your email id in the Android studio same as the one which you used to create firebase project.

5) Authenticate your login credentials.

6) Make sure the package name of the firebase project and the android application are same.

7) Make sure the name of firebase package is correct in the gradle.build file
Now lets configure our database authentication and storage setting.Enable the email id and password authentication method in sign in method tab. Their are various features provided by firebase for the developer. Also enable the google sign in as we need it if the used want to login with his/her google account.

For database create the data base in test mode. And done, we will be adding more rules for database in future as per requirement.

\section{Encryption}

Encryption is a method to transform the message or text in to unreadable format. encryption algorithm jumbles the message letters in such a way that it becomes no more understandable. To understand that message the reciever has to decode it again to transform it in to original form. Their are various way of encryption depending on the key type. For end to end encryption we need two keys, the private key and the public key. Working of E2EE in explained earlier. To see if our encryption method works or not we initially commented the encryption code and sent few message and then uncommented the code to see the encrypted code. following is the output of messages stored on the database before and after encryption.

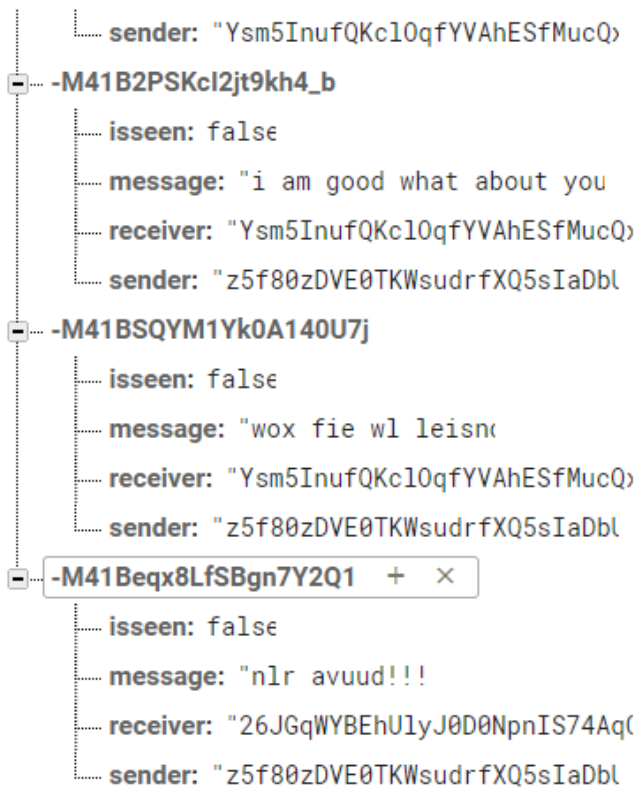

Fig. 2. Encrypted Chat in database

we can see how the encryption code encrypted the "Are you in canada" and "Hey buddy" in to unreadable encrypted form. while the message recieved by the receiver is in the original form.

How does it works?: So as we know if we encrypt a text we also need to decrypt that text. When the sender types the message and hits the send button normally it goes directly to the server, but here we take that code and encrypt it using the receivers public key. The encrypted text is stored in one variable is then sent to server. This prevent the hacker from 
getting the message. Even if the hacker get the message it would be in encrypted form. This encrypted message when received at receiver end gets decrypted automatically before getting display using that receivers private key. This private key is only stored in receivers phone.

\section{CONClusion}

In this paper, we introduced the working and implementation of encrypted chat application. How the messages are encrypted on device before moving to server. However, This application for now only deals with text messages, we are looking forward to encrypt the images before sending to server using computer vision techniques.The performance of the application over hundereds of users is not test yet as we have to create dummy user. Performance testing and image encrytion are in our list of future task.

\section{REFERENCES}

[1] Ali Makki Sagheer and Ammar Hammad Ali, "Design of Secure Chatting Application with End to End Encryption for Android Platform", Iraqi Journal for Computers and Informatics, 2017.

[2] https://developer.android.com/studio/write/firebase

[3] Akram R.N., Ko R.K.L. (2014) End-to-End Secure and Privacy Preserving Mobile Chat Application. In: Naccache D., Sauveron D. (eds) Information Security Theory and Practice. Securing the Internet of Things. WISTP 2014. Lecture Notes in Computer Science, vol 8501. Springer, Berlin, Heidelberg

[4] H.C. Chen and A.L.V. Epa, "A Rotation Session Key- Based Transposition Cryptosystem Scheme Applied to Mobile Text Chatting", Proceedings of The 28th IEEE International Conference on Advanced Information Networking and Applications (AINA2014), pp. 497 - 503, Victoria, Canada, May 2014.

[5] Chouhan, Kuldeep Singh and Srivaths Ravi. "Public Key Encryption Techniques Provide Extreme Secure Chat Environment." (2013). 